\title{
The use of interface workers to facilitate child and adolescent mental health services in primary care: a qualitative assessment
}

Mary Fox School of Psychology University of Exeter, Exeter, Devon, UK, Colin Greaves Mid-Devon Primary Care Research Group, Wyndham House Surgery, Silverton, Devon, UK and Jon Perry Child and Adolescent Family Services, Iddesleigh House, 97 Heavitree Road, Exeter, Devon, UK

\begin{abstract}
The need to improve the skills of primary care workers in the management of mildto-moderate mental health problems in children and adolescents is widely recognized. One model for providing such skills is the use of specialist psychiatric nurses as interface workers in a consultation-liaison role. This aims to provide training and expert advice to facilitate the detection, assessment and management of child and adolescent mental health problems. The objective of this study is to assess the impact of a pilot scheme which placed three consultation-liaison interface workers, for $15 \%$ of their time, in primary care. This study involved a qualitative assessment of health professionals' experiences of the new system, using constant comparison analysis of the transcribed texts of five focus groups. The setting was within the participating surgeries and at the secondary care centre. The participants comprised ten secondary care workers, ten GPs, seven health visitors and three school nurses. The main outcome measure was the views of the health care professionals of the interface worker service. The results showed that primary care staff reported strong, positive effects on confidence, morale and patient care, although a few possible problems were perceived in terms of carers' understanding of brief intervention. Prior to the introduction of the new system, they felt they had little to offer children and adolescents with mental health problems, but now they had a service to offer. The interface workers had particularly enhanced their treatment skills/options. However, the secondary care workers felt that there had been little effect on their workload although only four out of 54 practices feeding into them had had interface workers appointed. Although they recognized the potential benefits for mild to moderate cases in primary care, they were also concerned that scarce resources might be being directed towards lower priority cases. This pilot study concluded that this was an efficient, effective service offering a quick response to patients, equipping primary care workers with treatment skills and having a positive effect on the numbers of referrals to secondary care. Further, more extensive quantitative work is needed to find out if these conclusions are really justified.
\end{abstract}

Key words: mental health; child and adolescent; primary care; qualitative, interface

\section{Introduction}

Primary care is the setting for over $90 \%$ of patient interactions (DOH, 1998a), and is usually the first

Address for correspondence: $\bullet \bullet \bullet \bullet<$ aq1 $>\bullet$

E-mail: M.Fox@ex.ac.uk

(c) Arnold 2003
NHS contact point for child and adolescent mental health problems. However, primary care workers' skills in the detection and management of mental health problems are thought to be relatively poor (King, 1998). In addition, only a small proportion of cases identified by GPs (5-20\%) are referred to specialist psychiatric services (Goldberg and Jackson, 1992). While low referral rates to secondary 
services by GPs is part of a general trend, in this case reasons may include worries about the stigma attached to psychiatric care (Subostsky and Brown, 1990), lack of confidence in child psychiatric services and low awareness of the services (Chithiramohan et al., 1993). Even if a referral is made, there are often long waiting lists, and the main points of contact for children and adolescents during this period are health visitors, school nurses, and GPs.

The recent National Service Framework on Mental Health (DOH, 1999a), acknowledges that issues of both training and resources need to be addressed to improve care given at the primary level. While the white paper 'Our Healthier Nation' (DOH, 1998), highlights the fact that poor mental health in children and adolescents has increased over recent decades, and that 'early action in a child's life may improve their health and mental health in later life'.

One possible model for improving the primary care management of mental health problems is the consultation-liaison model of interface working. Here, secondary care specialists work to facilitate care at the primary level through providing training, and a source of expert advice to guide the assessment of cases, and the choice of care options (Gask et al., 1997). Consultation-liaison includes four main features:

- regular meetings between the primary care team and the interface worker;

- cases are only referred after joint discussion;

- some cases remain within primary care;

- feedback is given to the primary care team regarding referred cases.

Specific evidence on the use of consultationliaison in child and adolescent mental health problems is very thin. In particular, there is little research about the primary/secondary interface leading to service commissioning resulting from policy and theory rather than scientific evidence. A controlled implementation of interface workers in Portsmouth reduced referrals to secondary care by a third (Neira-Munoz and Ward, 1998). Primary care workers reported high satisfaction with the new service, and that their level of understanding of child mental health had increased.

In 1998, a pilot project was implemented in north and east Devon, placing three specialist child and adolescent clinical psychiatric nurses (CPNs) as interface workers in four practices, using a consultation-liaison model, this provided an opportunity to conduct some further research in this area.

\section{The service}

Using the consultation-liaison model outlined above the interface workers provided a service in which they:

- provided a training/advisory/consultation service to the primary care teams on treatment options and the suitability for referral;

- assessed cases and aided referral to secondary care;

- supported/supervised the primary care workers in visits to clients;

- took on cases briefly, for two or three sessions before handing over to the primary care teams.

This service took $15 \%$ of the CPNs' time, which was allocated on the basis of practice sizes. The remainder of their time was spent in secondary services.

This study aimed to examine the benefits and drawbacks of the new system, as perceived by primary and secondary care mental health workers. It was also intended to look for possible ways to improve the implementation and content of such a system.

\section{Methods}

\section{Design}

A qualitative investigation using five focus groups, was chosen to allow the open-ended exploration of the views across a variety of professions.

\section{Sample}

- Primary Care: Four surgeries were serviced by three interface workers. All GPs, health visitors and school nurses from these surgeries were invited to take part. The sample group all had experience of working with the interface workers, and represented a range of age, gender, professional training and experience in both rural and urban practices. The four group interviews contained seven (out of a possible nine), health visitors (forming two groups), a group of three school nurses (out of a possible five), and a group of ten GPs (out of a possible 15). 
- Secondary Care: This focus group consisted of three psychiatrists, three psychotherapists, three community psychiatric nurses and one occupational therapist.

\section{Procedure}

Interviews were conducted in the respective work places of the participants during normal working hours, using an independent researcher. The interviews followed a topic guide, which focused on extracting evaluations of the new system, as well as ideas for improving and developing the interface workers role, but participants were encouraged to talk in a relatively unconstrained way. All interviews were tape-recorded and transcribed.

\section{Analysis}

The constant comparative method of analysis was used to identify recurring themes and patterns within the transcription. This procedure draws categories and concepts from the data in an iterative process. The emerging themes are refined by splitting and merging, constantly checking the validity of the constructs with the raw data. The process continues until the constructs become saturated and an overarching core category develops which describes the story within the data (Strauss and Corbin, 1998).

\section{Results}

Speakers are identified in the tables as:

- HV: health visitors

- SN: school nurses

- GP: general practitioners

- SC: secondary care workers

Four main themes emerged from the analysis. These were perceived changes, the interface workers' role, mediating factors and problems and suggestions.

\section{Perceived Changes:}

Primary care workers evaluated the interface workers extremely positively, and the improved confidence of Health Visitors and School Nurses was particularly evident, as they now felt that they could effect change in peoples' circumstances that had not been possible before. The ability to offer effective and efficient help impacted on the job satisfaction of primary care workers as their frustrations were overcome by positive action. A small minority felt that the old system had been adequate. However, the vast majority told stories of a nonexistent service and long waiting lists.

\section{Changes in Referral:}

Changes in referral patterns were reported, with the interface workers being consulted in many mild to moderate cases. Previously primary care workers had done nothing, tried to cope within primary care, or waited months for a secondary appointment. This new service greatly speeded up treatment. There was a general feeling that the interface workers were enabling resolution of many cases, thus preventing the need for secondary referral. The other main reported change was that referrals to secondary care were being effected through the interface worker rather than through the GP. When referral to secondary care was necessary this process often ran more smoothly. However, some secondary care workers reported the referral process to be unchanged.

\section{Patient Benefits:}

Indications of the patient's perspective, were ascertained from the comments of primary care workers. The primary care workers reported that their clients felt they were getting a better service. Clients did not seem to feel that any mental health stigma was being attached by seeing the interface workers and families were delighted to have someone who could respond quickly to their needs. The benefits of more local availability of services were also recognised, especially in the more rural communities.

\section{Confidence:}

Increases in confidence and the feeling that skills had been enhanced were related to the effects of the teaching and support that the interface workers gave to primary care workers. Some felt that the main benefits were in terms of management skills and learning how to intervene with clients, rather than detecting problems. (See Table 1)

\section{Role of the Interface Worker Education:}

The interface workers were perceived as providing enormous support and giving extra education. 
Table 1 Perceived changes

We (now) actually try and deal with the problem, ..., make an intervention rather than it go away and get so bad you have to do something, .... when the child psychiatrist is not appropriate but sometimes is. GP

I think this satisfaction that children and families are having their needs meet more quickly and more satisfactorily ... SN

... you just see them again and again and again and in the process ...they will have been expelled from school and there's nothing you can do about that, it's hopeless. GP

It was a non-service wasn't it? GP

I refer more to her than I would have done to mental health. HV

In the old days $Z$ would say I'm not getting anywhere, could you make a referral? Well I don't think I've made a referral to secondary since last year. GP

She's been able to step in and get something sorted out quite quickly. She knows that part of the system. HV

I feel my skills have been enhanced ... its given me more confidence in dealing with things. HV

I don't dread them (mental health problems) quite as much. GP

I think we've always been able to identify problems, it's what to do with them when you've identified them. HV

Long may it continue. HV

The quality of the referrals is better and I'm sure that will have an impact on our work. SC

They were involved in teaching therapeutic skills, joint visits, putting forwards a different approach or affirming what was being done. General practitioners said that the interface workers' professional mental health insight was valuable in diagnosis of the source of problems, (e.g., Parent, child, school situation).

\section{Intervention:}

Some primary care workers found intervention the most useful aspect of the interface workers' role. According to primary care workers, the interface workers were also able to respond sometimes immediately or within a week or two, which was in stark contrast to the previous system. It was felt that the interface workers were fulfilling a role that no one else had been doing. If cases merited secondary care, then the interface workers acted as a referral point to the centralized services.

\section{Prevention:}

The interface worker system was judged to be arresting the development of problems by 'nipping them in the bud'. Although the effects of this were not yet being felt in secondary care it was thought that one day they would. (See Table 2)

\section{Mediating Factors}

Availability and workload:

The part-time nature of both interface and some primary care workers did not appear to create communication problems. Generally work-load had not increased and where it had this was thought worthwhile due to the satisfaction of offering the patients something at last.

\section{Understanding each others' roles:}

There was some disparity in the information given to primary care workers before the new service began. Some felt well prepared and were ready to use the system from the start, others did not really understand it. This led to some confusion about what the interface workers were doing, whether it was just a support and advice role, or whether it included intervention. The confusion was reflected in the different ways that the interface workers were used. It was felt that more 
Table 2 Role of the interface worker

She was particularly helpful ... saying this isn't the child's problem, it's a parental problem. That could wait 18 months before we came to that conclusion. GP

You call her when you're stuck and she either fixes it or. GP

...She knows a man who can. GP

The fact that we can't see an effect yet doesn't mean that the effect wouldn't be quite great after a while... I would expect to see if they were removed that ... we would have problems that would come up ... if someone doesn't go in and do something ... we get them and they are horrendous by then. SC

adequate preparation was needed for a successful launch.

\section{Reactions to the Interface Worker System:}

The few negative comments voiced came from one health visitor, who felt that the interface workers were too formal and sometimes acted inappropriately, (e.g., Suggesting more appropriate ways of parenting). This view was in stark contrast to the overwhelming majority who were full of praise both for the interface workers personally and the work they had done. There was a strong view that if the interface workers left they would leave a huge gap.

\section{Finance:}

Attitudes to financial outcomes were conflicting in terms of how well people thought the money put into the interface worker project had been spent. It was seen as a small resource that had a huge positive impact by both primary and secondary workers, but some secondary workers thought the money was misplaced in not supporting more severe cases. (See Table 3 )

\section{Problems and Suggestions:}

Introduction of the Interface Worker System:

One group of health visitors felt that they had not received an adequate introduction to the new system. This had led to misunderstandings about the role of the interface workers and a discontinuation of the service in one practice.

Suggested improvements to the Interface Worker System:

Suggestions from primary care workers included expanding the intervention role to that of an attached mental health worker and giving the primary care workers more protected time for Child and Adolescent Mental Health Services (CAMHS) work. Some secondary care workers thought that the system would work better if it was based in secondary care with the interface workers attached to particular surgeries.

Concerns about the future of the Interface Worker System:

Many secondary care workers felt that currently the interface workers were professionally isolated

Table 3 Mediating factors

Yes (workload increased) ... but there's more satisfaction when you feel your offering something at last to the school ... family ... it's a big bonus. SN

Sometimes I've thought this is more trouble than it's worth. HV

It was a fairly small resource ... look at all the difference it has made in all our lives. GP

If the resources were coming to us, those resources would have been spent more on higher up severity cases. It's hard to justify (preventative work) when they come in such numbers and severity. SC

It was useful to meet her ... she went through her role, ... that was very worthwhile. HV

I'm capable of assessing ... rather than go through a supervisory process. HV 
and would lose expertize if systems were not set up to counter this. They thought that the current arrangement was creating another tier unnecessarily, which made clinical governance harder to administer, as the interface workers were isolated from their secondary care colleagues and the systems being put in place.

\section{Disadvantages of the Interface Worker System:}

These concerns were voiced almost entirely by one person who felt that the new system slowed the process of referral up and was too formal. It had also been found that the short-term commitment to cases, the interface workers had been restricted to, caused some upset due to lack of continuity. The secondary care workers reported the frustrations of the practices without interface workers. ( Table 4)

\section{Discussion}

\section{Limitations}

While it can be argued that the results of qualitative data analysis are not generalizable to all situations, if rigorously carried out, their findings can be transferred to similar subject groups in similar contexts (Murphy et al., 1998). Thus, the results of this study should mirror the experience of other rural practices in the UK using a similar model of interface working.

\section{Primary care:}

The story that has emerged from the data is one of a group of primary care workers who have moved from a position of frustration to one of satisfaction. The frustration was caused by a system that 'wasn't really much of a system at all', that left its participants, both clinical and patient, in a state of impotence and dissatisfaction at the lack of service 'it's hopeless'. Problems were left unad- dressed to deepen until distress became so acute that 'you have to do something'. The effect this had on the primary care workers was to cause them to feel that they were letting patients down as all they had to offer was a 'nonservice'. While some had confidence in their abilities to identify mental health problems, others felt that their training had not equipped them to deal with these problems 'it's what to do with them (sic. the problems) when you've identified them'.

This uncomfortable position changed, with the introduction of the interface worker service, to one of satisfaction that they were at last able to offer their patients something 'that families are having their needs met more quickly and more satifactorily'. The effects the interface workers had were to reverse a culture of resignedly 'holding' to one of 'can do'. The support both in advice and training as well as intervention raised the levels of confidence, particularly of health visitors and school nurses as cases that previously would have been 'dusted under the carpet' were treated. They had the satisfaction of seeing new skills being effective, although, perhaps most satisfaction stemmed from seeing children and adolescents' problems arrested before they became a crisis. Mental health problems were 'no longer dreaded' as they had been before.

The preparation for the changing system was an issue that affected the attitudes of the primary care staff to the interface workers. Where this had been done well, with a clear exposition of who they were and their role, they had been fully accepted, 'it was useful to meet her...she went through her role... that was very worthwhile'. Where there was a lack of formal preparation (in one particular group), this appears to have been an unfortunate precursor to a breakdown in relationships. That these particular primary care workers were not clear what the role of the interface worker was, possibly led to feelings that the interface workers

Table 4 Problems and suggestions

There are potential risks then, in terms of them becoming isolated perhaps by idiosyncratic friends without the quality control that you get from Clinical Governance system throughout the service. SC

The frustrations of the practices that haven't got them has been increased ... they don't understand it's a project ... and want to know why they haven't got one too. SC 
had been brought in because they were not good enough at mental health issues 'I'm capable of assessing...rather than go through a supervisory process'. This may have been combined with a belief that the treatment of mental health was not within their remit, as they had not been trained for it. Therefore they were being implicitly criticized for failing to do something that was not perceived as being part of their job. Clarification of the role of the interface workers was also necessary due to the preference of some primary care workers for the interface workers to take on cases rather than improve their skills. The main thrust of the consultation-liaison model is for the CPNs to provide training and advice, supporting the primary care workers as their expertise improves. This issue needs addressing in the development of future services.

\section{Secondary Care:}

In contrast to the above, some secondary care workers felt that the process of referral was unchanged. This may be because only four of the 54 practices feeding into the CAMHS were using interface workers. The secondary care workers were therefore feeling little effect from the interface workers' presence. However, an improvement in the quality of referrals from some of the practices involved was recognised, 'The quality of the referrals is better and I'm sure that will have an impact on our work'. While the benefits in primary care were acknowledged, there was some resentment that the money had been found to tackle mild to moderate mental health in the community while they were left with the same inadequate budget to deal with an over-burdened case load of severe problems it's hard to justify (this allocation of resources)'.

This sentiment echoes Gournay and Brooking (1995), who describe CPNs working in primary care as 'expensive luxuries' whose employment is to the detriment of people with serious and enduring mental health problems, although these views relate to adult services and may not apply to CAMHS. It was beyond the scope of this project to economically evaluate the interface workers; however, the secondary care workers themselves acknowledged that if the small amount of money that had gone into the interface worker project had gone into secondary care it would have 'gone, just disappeared'. The view that sees interface workers in primary care as luxury items also ignores the possible longer-term preventative nature of the interface workers' role with its attendant economic and social benefits in years to come, and possibly the shorter term benefits of better 'care-taking' of clients on waiting lists, so that the severity of their illness when they are finally seen in secondary care may be much less than if the previous 'nonexistent' primary care system had been applied.

The secondary care workers may have felt that putting the emphasis on primary care undermined their more specialist expertise. This unconvinced attitude highlighted the polarity of the system. Cases were either dealt with in the community without input from secondary care or handed over to secondary care and no longer the responsibility of primary care. This typifies the problem of the primary-secondary care interface, whereby there is a false boundary constructed between the two care systems, and little continuity of care across this boundary.

The interface worker scheme seems to be highly acceptable within primary care, and appears to improve primary care worker confidence and skills in dealing with child and adolescent mental illness. It may be that focusing resources at the interface prevents a number of mild to moderate cases from becoming more severe, and it is likely that patients will be more responsive to treatment at this stage. However, the scheme must also be assessed in terms of the detraction of resources from dealing with higher severity illness. It is particularly recommended therefore that research is conducted to weigh these potential costs against the potential benefits of earlier intervention, prevention, and better 'care-taking' of waiting list clients offered by the interface worker model.

Secondary care workers considered that the interface workers were isolated in the community and were concerned that their level of expertise would diminish over time as they lost contact with mental health professionals. This concern is shared by the government in the Health Service Circular (HSC) 1999/126, which calls for 'sufficient trained CAMHS professionals with an appropriate range of skills organised in a manner that avoids professional isolation.' (DOH, 1999b).

This study reflects the findings of the Portsmouth study that GPs and health visitors reported high levels of satisfaction with the liaison service, as well as a decrease in referrals to secondary care 
(Neira-Munoz and Ward, 1998). However, in contrast to the Portsmouth study, this study found that the primary care workers believed that their skills in managing mental health problems had increased. This may reflect differences in the job descriptions of the interface workers. In this study, the workers had a clearly defined role to educate and facilitate primary care workers, so that they could deal with more of the subclinical caseload themselves, rather than to simply act as an in-house referral resource. The interface worker service was found to be acceptable to primary care workers, and seemed highly effective in increasing confidence and skills in treating mental health problems in children and adolescents. Future implementation might be facilitated by an improved introduction for the GP practices, systematic secondary care supervision, a clear agreement of roles and protected time for the primary care staffs' training.

As a result of the success of the pilot scheme, the interface worker service has been extended to the rest of east Devon practices. The service now needs wider assessment to establish cost-effectiveness, the sustainability of the service, the views of the interface workers and the users and to determine longer-term outcomes on both patterns of service use, and patient health and satisfaction.

\section{References}

Chithiramohan, R. N., Ballard, C. G., Baxter, M. A., Jones, L., Handy, S., McGibben, L., Sheikh, A. and Silveira, R. 1993:
Factors influencing general practitioner referral to a child psychiatric service. Irish Journal of Psychological Medicine $10,144-7$.

Department of Health. 1998: Our Healthier Nation. London: HMSO.

Department of Health. 1998a: $R \& D$ in primary care. National Working Group report. London: HMSO.

Department of Health. 1999a: National Service Framework for Mental Health. London: HMSO.

Department of Health 1999b: HSC/LAC NHS Modernisation Fund and Mental Health Grant for Child and Adolescent Mental Health Services. London: HMSO.

Gask, L., Sibbald, B. and Creed, F. 1997: Evaluating models of working at the interface between mental health services and primary care. British Journal of Psychiatry 170, 6-11.

Goldberg, D. and Jackson, G. 1992: Interface between primary care and specialist mental-health care. British Journal of General Practice 42, 267-9.

Gournay, K. and Brooking, J. 1995: The community psychiatric nurse in primary care - an economic analysis. Journal of Advanced Nursing 22, 769-78.

King, M. 1998: Mental health research in general practice. British Journal of General Practice 48, 1295-7.

Murphy, E., Dingwall, R., Greatbatch, D., Parker, S. and Watson, P. 1998: Qualitative research methods in health technology assessment: a review of the literature. Alton: Health Technology Assessment.

Neira-Munoz, E. and Ward, D. 1998: Side by Side. Health Service Journal 26-7.

Strauss, A. and Corbin, J. 1998: Basics of Qualitative Research. London: Sage

Subostsky, F. and Brown, R. M. 1990: Working alongside the general practitioner. Childcare, Health and Development 16, 189-96. 